\title{
Cochlear Model for the Evaluation of MRI Sequences and Cochlear Implant Electrode Pattern at 3T
}

\author{
Lars Uwe Scholtz ${ }^{1}$ Conrad Riemann ${ }^{1}$ Hans Björn Gehl ${ }^{2}$ Holger Sudhoff ${ }^{1}$ Ingo Todt ${ }^{1}$ \\ ${ }^{1}$ Department of Otolaryngology, Head and Neck Surgery, Medical \\ School OWL, Bielefeld University, Campus Mitte, Klinikum \\ Bielefeld, Germany \\ 2Department of Radiology, Bielefeld University, Klinikum Bielefeld, \\ Germany \\ Address for correspondence Ingo Todt, PD Dr. Med, Department \\ of Otolaryngology, Head and Neck Surgery, Medical School OWL, \\ Bielefeld University, Campus Klinikum Bielefeld, Teutoburgerstr. 50, \\ 33604 Bielefeld, Germany (e-mail: todt@gmx.net).
}

Ann Otol Neurotol ISO 2021;4:6-10.

\begin{abstract}
Keywords

- MRI

- cochlear implant

- cochlear model

- electrode position

- MRI sequences
\end{abstract}

Introduction Cochlear implant (Cl) magnets and surgical techniques (e.g., positioning) have made an impact on the relationship between $\mathrm{Cl}$ and magnetic resonance imaging (MRI) by solving the problem of pain and artifact. Recent investigations displayed the possibility to evaluate the $\mathrm{Cl}$ electrode position by MRI in vivo. However, further improved perceptual quality is needed to allow an improved evaluation of the electrode.

Aims The aim of this study was to assess a cochlear model for the examination of $\mathrm{Cl}$ electrode pattern and MRI sequences in vitro.

Materials and Methods Weinvestigated Clelectrodes in a fluid-filled three-dimensional artificial scala tympani model combined with a fluid package in a $3 T$ MRI scanner. Different high-resolution $\mathrm{T} 2$ sequences $(0.6-0.2 \mathrm{~mm}$ voxel size) were used for the visual electrode pattern evaluation for finding an optimized sequence.

Results Artificial models can be used to evaluate MRI characteristics of $\mathrm{Cl}$ electrodes. In our scanner configuration, a $0.3 \mathrm{~mm}$ voxel and $0.9 \mathrm{~mm}$ slice thickness sequence showed the best compromise between resolution and scanning time.

Conclusion and Significance MRI model-based testing can be performed in vitro to evaluate $\mathrm{Cl}$ electrodes' pattern and to optimize sequences. An MRI model is a tool for in vitro testing of MRI sequences and might help for future in vivo applications.

\section{Introduction}

Second generation of cochlear implant $(\mathrm{CI})$ magnets and improved surgical techniques (e.g., implant positioning) have made a significant impact on the relationship between a CI and magnetic resonance imaging (MRI) scanning by solving the problem of pain inside the scanner ${ }^{1}$ and cochlear/internal auditory canal obscuring artifacts. MRI has changed from a contraindication to a diagnostic tool. ${ }^{2}$ Recent publications showed the opportunity to evaluate the $\mathrm{Cl}$ electrode position by MRI in vivo and underline that the magnet artifact is a solved problem by specific implant positioning, head positioning, and choice of sequence. ${ }^{2-4}$

In vitro testing allows the transfer of specific information to improve the in vivo situation. Models or in vitro observation in temporal bones has been used for the computed tomography (CT) evaluation of electrode position, ${ }^{5}$ the estimation of force behavior during insertion, ${ }^{6}$ and evaluation of intracochlear pressure during insertion in temporal bones $^{7}$ and different models. ${ }^{8}$
Published online September 21, 2021
DOI https://doi.org/ 10.1055/s-0041-1735992 ISSN 2581-9607 (c) 2021. Indian Society of Otology.

This is an open access article published by Thieme under the terms of the Creative Commons Attribution-NonDerivative-NonCommercial-License, permitting copying and reproduction so long as the original work is given appropriate credit. Contents may not be used for commercial purposes, or adapted, remixed, transformed or built upon. (https://creativecommons.org/licenses/by-nc-nd/4.0/).

Thieme Medical and Scientific Publishers Pvt. Ltd. A-12, 2nd Floor, Sector 2, Noida-201301 UP, India 
The use of a model can be assumed to be beneficial as a tool to visually understand the electrode pattern in MRI scanning. It has multiple advantages in comparison to a direct evaluation and comparison of different MRI sequences in humans. ${ }^{9,10}$ Initial in vivo observations of the MRI patterns of $\mathrm{CI}$ electrodes showed general differences and characteristics in terms of resolution and quality of scans, requiring further improvement of MRI sequences. ${ }^{11}$

The major advantage of a model is the application of multiple scans with different sequence parameters without any patient burden. The purpose of MRI sequences evaluation is to optimize its resolution, voxel size, and duration. This process can be extremely time consuming. The introduction of MRI scans as postoperative tool for the evaluation of $\mathrm{CI}$ patients requires a balanced sequence showing a good compromise between resolution, scanning time, and slice thickness. An independent model seems necessary as various MRI scanner suppliers differ substantially in terms of their parameters.

The visual pattern of $\mathrm{CI}$ electrode is different from a CT pattern in terms of the fluid diminishing effect in contrast to an artifact visualization in a deep vein thrombosis/computed tomographic scan. This visual change needs to be studied to get familiarized. This can be easily accomplished by a model simulating different positional situations and electrodes.

Focusing on the cochlea, an electrode position (scala position) in the axial plane can be evaluated even in the MRI. ${ }^{3}$ The visual pattern in the coronal plane is more difficult to recognize as small changes may lead to torsional visual changes.

MRI scanning of models or temporal bones-different to CTs-requires a proper fluid signal. The attempt to scan an area with a low fluid signal results in an interruption of the MRI scanner procedure. ${ }^{12}$ As an electrode evaluation is only possible in a T2 sequence detecting fluid areas, this problem needs to be solved.

The goal of this study was to establish a technique for the in vitro MRI evaluation of $\mathrm{CI}$ electrodes and determine optimized sequences.

\section{Materials and Methods}

A three-dimensional (3D)-printed plastic scala tympani model of the cochlea (-Figs. 1 and $\mathbf{2}$ ) was filled with distilled water and inserted with different CI electrodes (Advanced Bionics, Stäfa, Swiss: HFMS; Cochlear, Melbourne, Australia: Contour, x32, Slim straight, Medel, Innsbruck, Austria Flex 28; Oticon, Vallauris, France: EVO). The design of each electrode can be classified into two groups (perimodiolar vs. lateral). The information for each electrode is additionally added to the legends. The model was provided by a CI manufacturer. This model was sealed to prevent evaporation. Afterward, the model was placed in a regular available gel-filled cool pack. This gel-filled cool pack was wrapped around a 51 MRI scanning normation bottle ( - Figs. 3 and $\mathbf{4}$ ). Normation bottles are used in every radiologic department to perform normation tasks. The bottle was placed in the 3T MRI scanner (Philips Acura) and different T2 2D drive multishot sequences were performed (-Table 1). In all cases, a coronal plane was performed for the scanning.

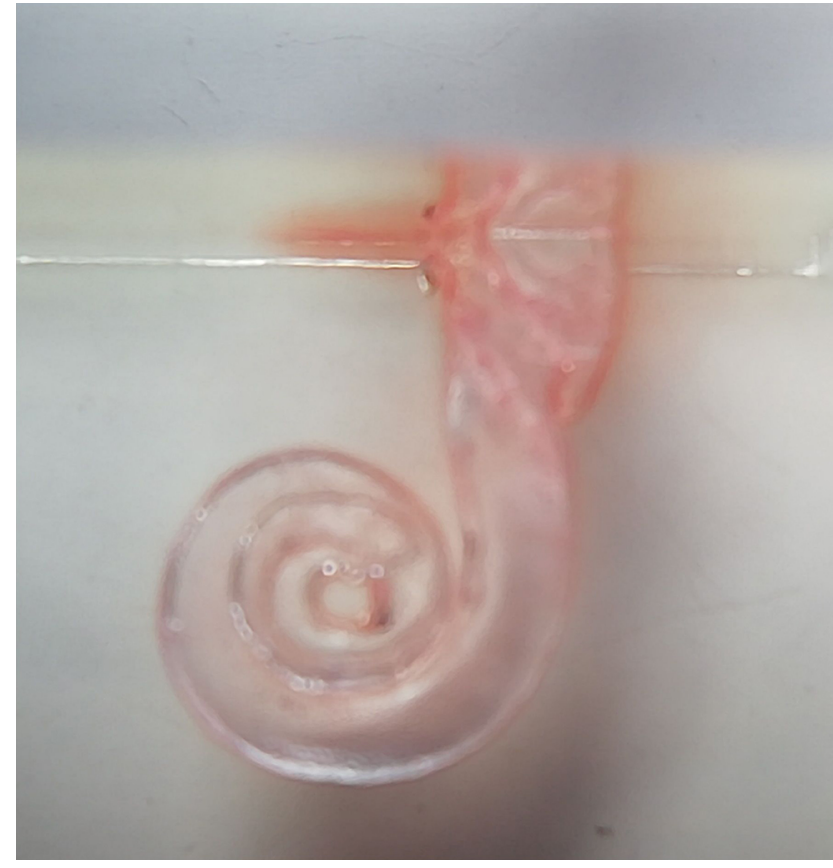

Fig. 1 Three-dimensional-printed scala tympani model of the cochlea.

\section{Results}

The 3D print of a cochlea enables the wrapping of a gel-filled cool pack around the model. This high grade of fluid in the cool pack and the contrasted fluid of the artificial 3D model offered an excellent contrast for the evaluation. The block configuration of the model allowed an easy angulation during the scanning survey.

The different electrodes were distinguishable and exhibited characteristic patterns. Lateral wall electrodes showed a T2 signal medially to the implant body and differentiated from each other by their number of contacts. This led to an identification of EVO (-Fig. 5), Flex 28 (-Fig. 6A-C), and slim straight array ( $\mathbf{- F i g}$. 7A, B). The pattern of modiolar

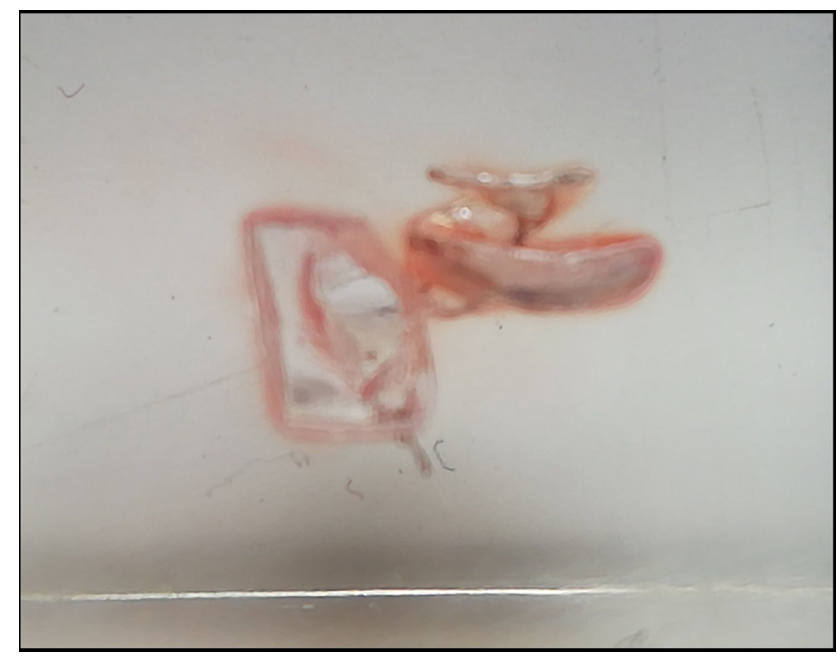

Fig. 2 Three-dimensional-printed scala tympani model of the cochlea. 
electrodes showed a fluid signal both medially and laterally from the electrode for the contour ( - Fig. 8A, B) and the HFMS electrode ( - Fig. 9A, B). Interestingly, the x32 device displayed only a fluid signal laterally, due to the tight wrapping of its electrode ( - Fig. 10).

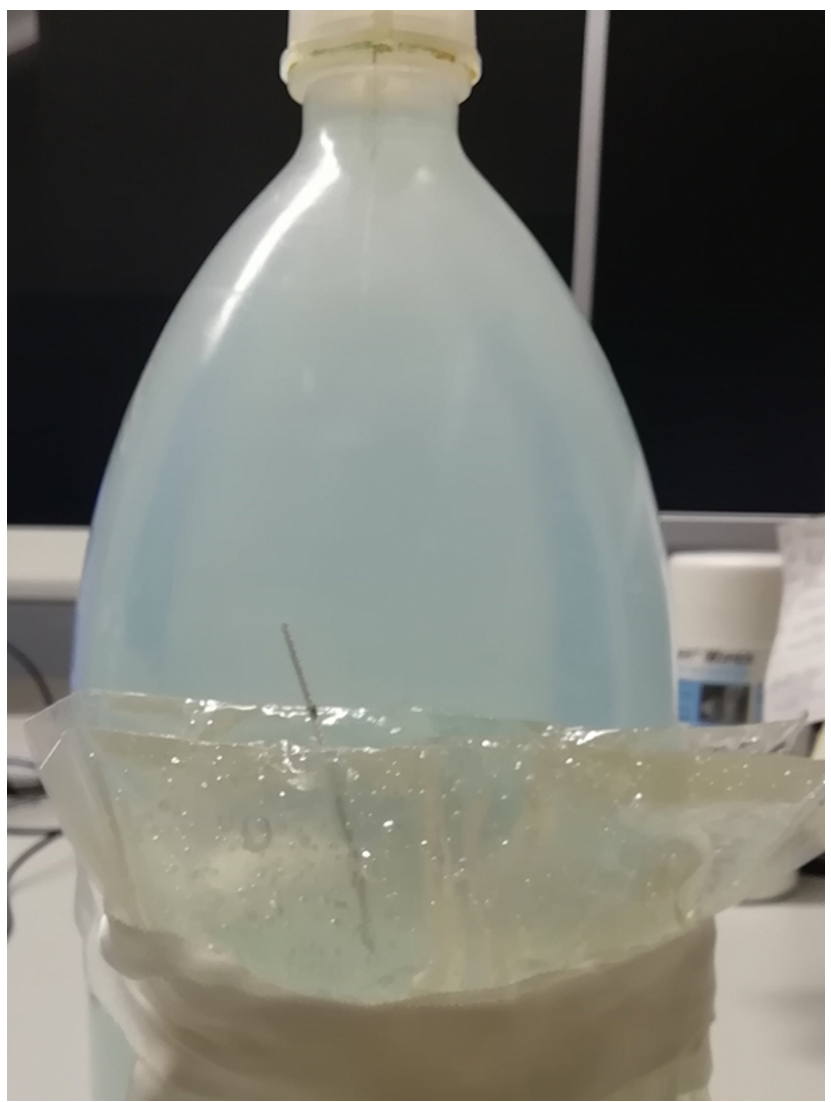

Fig. 3 Model attached wrapped with a cool pack around a Normation bottle.

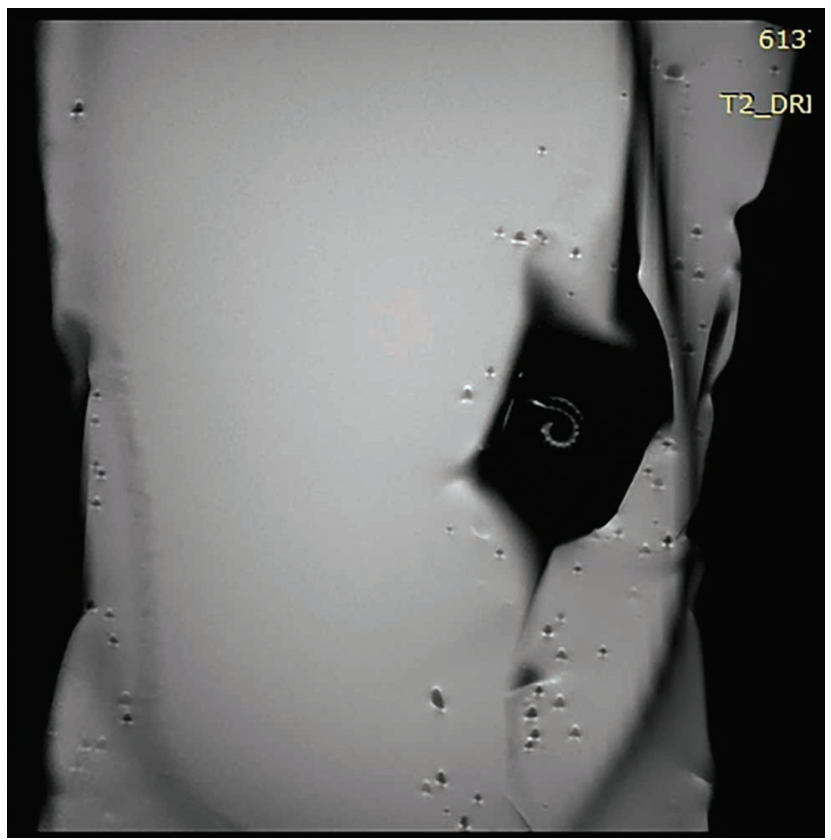

Fig. 4 Model attached wrapped with a cool pack around a Normation bottle.

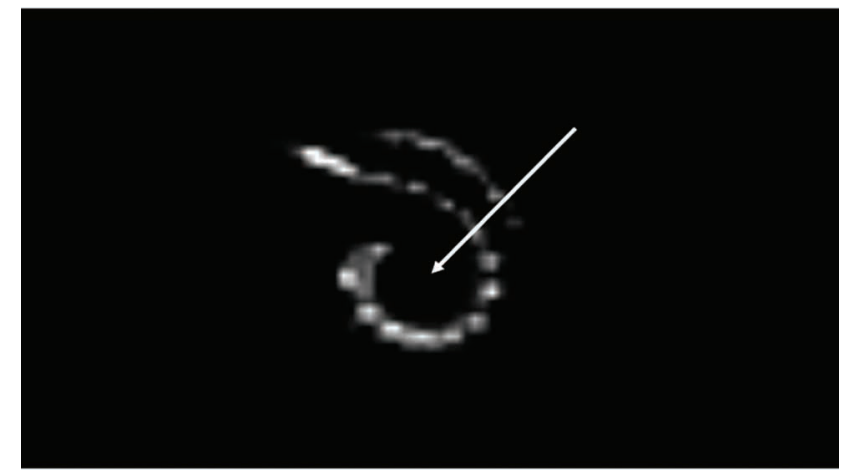

Fig. 5 Oticon EVO electrode (lateral) magnetic resonance imaging scan with Sequence 2. An arrow indicates the modiolus position.
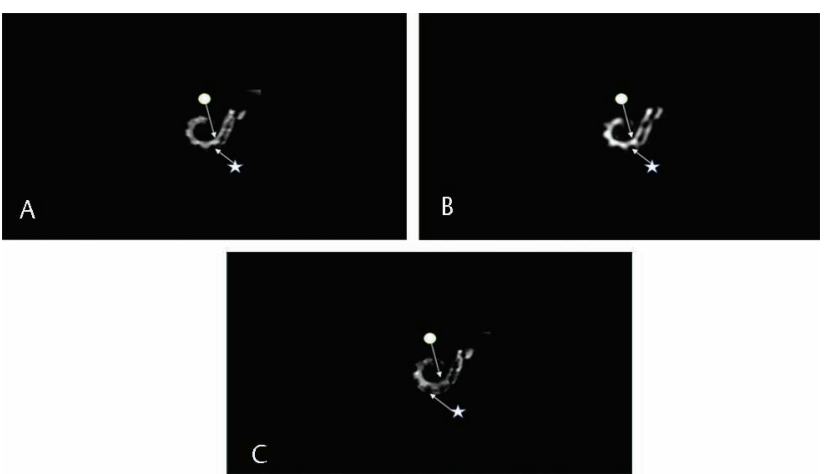

Fig. 6 (A-C) MED-EL Flex 28 electrode (lateral) at magnetic resonance imaging scan with Sequences 1,4 , and 3 . Star indicates electrode, and ball indicates fluid signal.
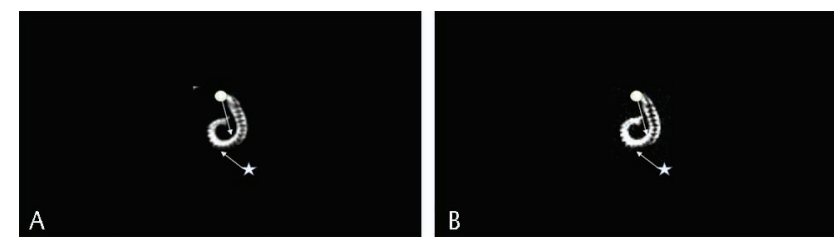

Fig. 7 (A, B) Cochlear slim straight electrode (lateral) at magnetic resonance imaging scan with Sequences 2 and 1 . Star indicates electrode, and ball indicates fluid signal.
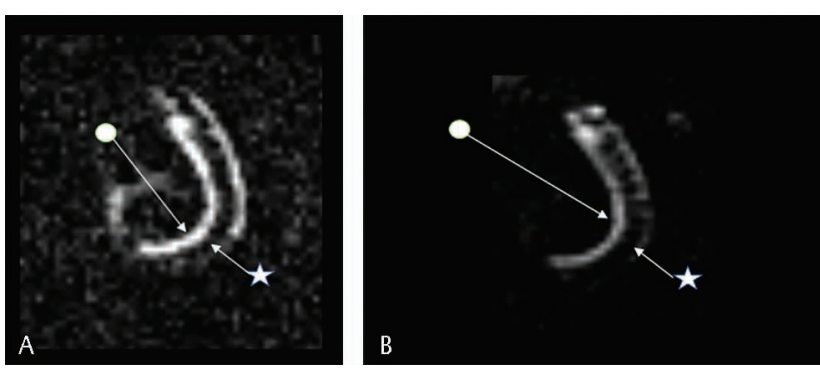

Fig. 8 (A, B) Cochlear contour electrode (perimodiolar) at magnetic resonance imaging scan with Sequences 1 and 2 . Star indicates electrode, and ball indicates fluid signal.

Additionally, visible contacts allowed a differentiation between contour and HFMS electrodes. The evaluation of the different applied sequences highlighted the importance of adjusting following parameters: slice thickness, resolution, 

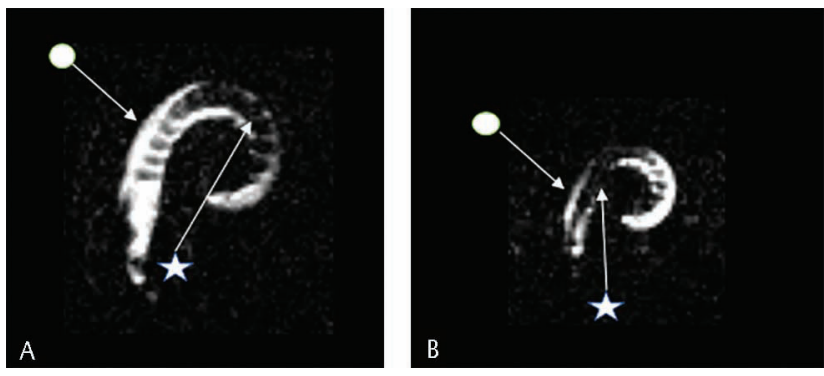

Fig. 9 (A, B) Advanced Bionics HFMS electrode (modiolar) at magnetic resonance imaging scan with Sequences 3 and 1 . Star indicates electrode, and ball indicates fluid signal.

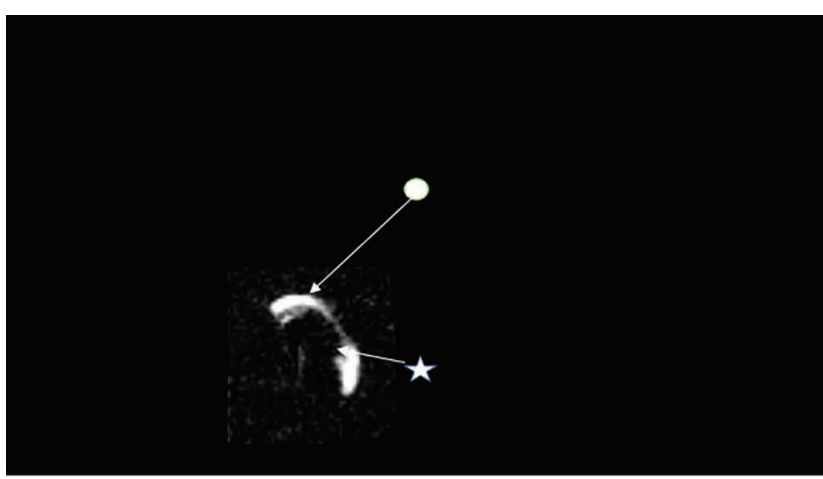

Fig. 10 Cochlear $\times 32$ electrode (perimodiolar) at magnetic resonance imaging scan with Sequence 1. Star indicates electrode, and ball indicates fluid signal. and scanning time. The choice of the different sequences followed the aim of finding a good compromise between these three parameters. These parameters are interdependent and determined by the scanner. Comparing Sequence 1 with 4 for the Flex 28 electrode indicated that contrasting is more important for the differentiation of electrode structures than slice thickness. The same comparison for the HFMS and Flex 28 electrodes revealed that an increase in resolution from $0.3 \times 0.3 \mathrm{~mm}$ to $0.2 \times 0.2 \mathrm{~mm}$. However, the improved resolution prolonged the scanning time from 16 minutes to 38 minutes.

\section{Discussion}

MRI scanning of $\mathrm{CI}$ electrodes in a model is a complex method. The presented model allowed in vitro testing of sequences prior to the introduction a clinical tool. It prevented significant discomfort for the patients avoiding repetitive testing and long scanning periods. The addition of the application of a cool pack to the 3D artificial model solved the general problem of a fluid contrast, which is needed for the MRI scanner not to interrupt the procedure. ${ }^{12}$

Using a model for the evaluation of electrodes makes the testing of MRI sequences independent from patients. This technique provides an easy way to evaluate and optimize sequences and electrodes for the MRI. A comparison of two different sequences indicated the value of a model for the

Table $1 \mathrm{MRI}$ description of the different MRI sequence parameter with evaluated electrodes (The different sequences are used as numbered).

\begin{tabular}{|c|c|c|c|c|c|c|c|c|c|c|c|}
\hline Electrodes & Contour & Contour & Hi Focus & Flex 28 & Straight & Straight & $\begin{array}{l}\text { Hi } \\
\text { Focus }\end{array}$ & $\begin{array}{l}\text { Oticon } \\
\text { evo }\end{array}$ & Flex 28 & Flex 28 & X32 \\
\hline Fig. & $8 a$ & $8 b$ & $10 a$ & $6 b$ & $7 \mathrm{~b}$ & $7 a$ & $10 \mathrm{~b}$ & 5 & $6 c$ & $6 a$ & 9 \\
\hline Sequence & 1 & 2 & 3 & 4 & 1 & 2 & 1 & 2 & 3 & 1 & 1 \\
\hline $\begin{array}{l}\text { Slice thick- } \\
\text { ness (mm) }\end{array}$ & 0.9 & 2 & 1.4 & 0.5 & & & & & & & \\
\hline $\begin{array}{l}\text { Resolution } \\
(\mathrm{mm})\end{array}$ & $0.3 \times 0.3$ & $0.3 \times 0.3$ & $0.2 \times 0.2$ & $0.6 \times 0.6$ & & & & & & & \\
\hline FOV & $150 \times 150$ & $150 \times 150$ & $150 \times 150$ & $150 \times 150$ & & & & & & & \\
\hline Time (min) & 16 & 17 & 38 & 13 & & & & & & & \\
\hline $\mathrm{TE}$ (ms) & 100 & 87 & 87 & 87 & & & & & & & \\
\hline TR & 3000 & 3000 & 3000 & 3000 & & & & & & & \\
\hline TSE Tact & 17 & 16 & 11 & 16 & & & & & & & \\
\hline Flip angle & $90^{\circ}$ & $90^{\circ}$ & $90^{\circ}$ & $90^{\circ}$ & & & & & & & \\
\hline $\begin{array}{l}\text { Refocus } \\
\text { control }\end{array}$ & 120 & 120 & 120 & 120 & & & & & & & \\
\hline Metric & 512 & 512 & 512 & 512 & & & & & & & \\
\hline NSA & 5 & 5 & 5 & 8 & & & & & & & \\
\hline $\begin{array}{l}\text { Foldover } \\
\text { direct }\end{array}$ & AP & AP & AP & AP & & & & & & & \\
\hline
\end{tabular}


refinement of a high-resolution MRI sequence. The comparison illustrates that the voxel size is highly important for the quality of visualization ( $\boldsymbol{- \text { Fig. }} \mathbf{6 B}, \mathbf{C}$ ). Investigating the same electrode ( - Fig. 6B; slice thickness/resolution $0.5 \mathrm{~mm}$, voxel size $0.6 \times 0.6 \mathrm{~mm}$ [Sequence 4]) shows less contoured edges than ( - Fig. 6A; slice thickness/resolution $0.9 \mathrm{~mm}$, voxel size $0.3 \times 0.3 \mathrm{~mm}$ [Sequence 1]). The lower resolution is a disadvantage of Sequence 4 in comparison to Sequence 3. A combination of both preferred parameters is not possible as a physical conflict of the MRI scanner would occur. Finally Sequence 1 was preferred since it was the best compromise between the three parameters slice thickness, resolution (voxel size), and scanning time (-Table 1).

Additionally, the scan of different electrodes can be performed to gain an improved understanding of the complex visual MRI pattern changes, which are associated with changes of the model position in the coronal plain.

Evaluation of electrode position in the axial plain with an adequate resolution is applicable even with a $1.5 \mathrm{~T}$ MRI scanner. ${ }^{3}$ Understanding the coronal visual pattern is an important step for the evaluation of insertional depth by MRI. Comparison of the electrodes shows a general difference between lateral wall electrodes and perimodiolar/modiolar electrodes. The fluid signal of lateral wall electrodes is medially positioned. Contrary, we detected a laterally positioned fluid signal in perimodiolar/modiolar electrodes. It enables easy differentiation between the lateral wall electrode types by counting the number of contacts (-Figs. 6 and 7). A differentiation of perimodiolar/modiolar electrode types was possible. The $\mathrm{x} 2$ electrodes revealed no medial fluid signal, while the contour and HFMS electrodes were characterized by medial and lateral fluid signals.

The evaluation of $\mathrm{CI}$ electrodes in temporal bones by MRI is challenging. Related to the complex aeriation, limitations due to the requirement of an air bubble-free cochlea and a fluid contrast for the MRI scanning persist. The limitations of this study are related to the model. The employed model is only a scala tympani print. Ideally, a scala vestibuli integration into the model is required for a complete evaluation of the electrode MRI pattern. The possible translocation of electrodes cannot be simulated in this model. Alternatively, specific temporal bone models might be generated, which solve the problem of a so far missing fluid contrast. A further evaluation of the elaborated sequences in vivo is necessary.

\section{Conclusion}

MRI testing can be performed in vitro for the evaluation of $\mathrm{CI}$ electrode patterns and optimization of sequences. The application of this model allowed the evaluation of MRI sequences and scanner characteristics.

\section{Statement of Ethics}

The data used to support this study's findings are available from the corresponding author upon request.
Data Availability Statement

All data are implemented in the manuscript.

\section{Authors' Contributions}

Todt has conceptualized and written the manuscript, while H. Sudhoff, C. Riemann, and H.B. Gehl have cowritten the manuscript.

\section{Conflict of Interest}

None.

\section{Acknowledgment}

We would like to thank S. Trees for her helpful contribution to the experiments.

\section{References}

1 Kim BG, Kim JW, Park JJ, Kim SH, Kim HN, Choi JY. Adverse events and discomfort during magnetic resonance imaging in cochlear implant recipients. JAMA Otolaryngol Head Neck Surg 2015;141(1):45-52

2 Walton J, Donnelly NP, Tam YC, et al. MRI without magnet removal in neurofibromatosis type 2 patients with cochlear and auditory brainstem implants. Otol Neurotol 2014;35(5):821-825

3 Stratmann A, Mittmann P, Rademacher G, et al. MRI-based estimation of scalar cochlear-implant electrode position. BioMed Res Int 2017;2017:6372704

4 Tek F, MüLler S, Boga E, et al. 3T MRI-based estimation of scalar cochlear implant electrode position. Acta Otorhinolaryngol Ital 2019;39(4):269-273

5 Bartling SH, Gupta R, Torkos A, et al. Flat-panel volume computed tomography for cochlear implant electrode array examination in isolated temporal bone specimens. Otol Neurotol 2006;27(4):491-498

6 Roland JT Jr. A model for cochlear implant electrode insertion and force evaluation: results with a new electrode design and insertion technique. Laryngoscope 2005;115(8):1325-1339

7 Greene NT, Mattingly JK, Banakis Hartl RM, Tollin DJ, Cass SP. Intracochlear pressure transients during cochlear implant electrode insertion. Otol Neurotol 2016;37(10):1541-1548

8 Todt I, Mittmann P, Ernst A. Intracochlear fluid pressure changes related to the insertional speed of a $\mathrm{CI}$ electrode. BioMed Res Int 2014;2014:50724110.1155/2014/507241

9 Carlson ML, Neff BA, Link MJ, et al. Magnetic resonance imaging with cochlear implant magnet in place: safety and imaging quality. Otol Neurotol 2015;36(6):965-971

10 Edmonson HA, Carlson ML, Patton AC, Watson RE. MR imaging and cochlear implants with retained internal magnets: reducing artifacts near highly inhomogeneous magnetic fields. Radiographics 2018;38(1):94-106

11 Sudhoff H, Riemann C, Gehl HB, et al. MRI pattern of various cochlear implant electrodes in vivo Ann Otol Neurotol; 2020 doi:10.1055/s-0040-1708796

12 Scholtz LU, Gehl HB, Sudhoff H, et al. Estimation of different cochlear implant electrodes position in the human temporal bone by high resolution 3T MRI, LRO, 2018. Meeting of the German ENT Society, Erfurt. doi:10.1055/s-0038-1640587 